\title{
Novel and non-toxic treatment for night terrors
}

\author{
Bryan Lask
}

Department of

Psychological Medicine, Great Ormond Street

Hospital for Sick Children, London WC1N 3JH

Bryan Lask, FRCPSYCH, consultant psychiatrist
Night terrors (pavor nocturnus) are characterised by screaming, extreme distress, autonomic arousal, and increased body movements (sometimes including sleepwalking).' They affect about $3 \%$ of children, often occurring every night. The episodes arise in slow wave sleep (stages 3 and 4), most commonly within two hours of falling asleep.' ' The child cannot be comforted or woken and has no memory of the episodes the next day. There is rarely evidence of an associated psychiatric disorder, and the attacks are thought to represent a fault in slow wave sleep. ${ }^{\prime}$

A wide range of drugs has been suggested as treatment, including bromazepam, diazepam, midazolam, and imipramine. ${ }^{3.5}$ As there is no firm evidence that they are effective and potential side effects must be a cause for concern non-pharmacological measures should be tried first. Behavioural alteration of the sleep pattern has not previously been described.

\section{Patients, methods, and results}

Nineteen successive children (age range 5-13, mean $8 \cdot 7,11$ boys eight girls) referred to the department of psychological medicine for treatment of night terrors were found to have no physical abnormalities and no psychiatric disorder. Electroencephalograhic recordings either yielded normal results or showed nonspecific changes. All except one of the children were of average or above average intelligence. The terrors had lasted for between four and 37 months, with a mean of eight months, and generally occurred four to five nights a week.

Each child was treated as follows: parents were asked to note for five successive nights at what time the episodes occurred, and whether there were signs of autonomic arousal, such as sweating, tachycardia, or increased movements. They were then instructed to wake their child fully 10-15 minutes before the terror occurred or, if this was too difficult to specify, when autonomic arousal was noted. After four to five minutes the child was allowed to return to sleep. The parents were told to stop waking up their children when the terrors had stopped. No other instructions were given.

In each case the night terrors stopped within a week of starting treatment. In three cases terrors returned four to seven weeks later but were completely eliminated by restoration of treatment for a further week. At follow up one year later there had been no further relapses. One boy aged 9 had developed intermittent headaches for which no organic cause could be found but which were eliminated by relaxation twice a day. A girl of 11 had developed a phobia about school four months after treatment was started. This was satisfactorily treated with the help of her parents in a gradual return to school over three weeks. There was no evidence of psychological disturbance or of development of other symptoms in the other children.

\section{Comment}

Although the condition is benign and usually resolves within a few years, treatment is indicated because of the intense distress experienced by parents and children and the dangers of sleepwalking. The successful elimination of night terrors by behavioural alteration of the sleep pattern shows that treatment with drugs, which has not been proved to be effective, is unnecessary. Interruption of faulty slow wave sleep seems to cause reversion to a normal sleep pattern and resolution of the disorder. This technique may also be used for sleepwalking alone.

\footnotetext{
Matthews B, ()akey M. Triumph over terror. Br Med f 1986;292:203.

2 Fenwick P. Murdering while asleep. Br Med f 1986;293:574-5.

3 Vela A, Doblade\% M, Ramos M, et al. Action of bromazepam on sleep of children with night terrors. Pharmatherapeutica 1982;3:247-58.

4 Popoviciu L Corfariu O. Efficacy and safety of midazolam in the treatment of night terrors in children. Br f Clin Pharmacol 1983:16:975-1025.

W'eissbluth $M$. Is drug treatment of night terror warranted? Am 7 D) is (hild 1984:138: 1087.

Accepted 28. April 1988
}

\section{Severe reaction to diphosphonate: implications for treatment of Paget's disease}

\author{
A T Elliott, T Murray, R M Mackie, \\ J A Hunter
}

We report a potentially life threatening allergic reaction to sodium medronate, a component of the radiopharmaceutical most widely used for bone scanning. Sodium medronate is a diphosphonate, congeners of which are being used to treat Paget's disease.

\section{Case report}

A 47 year old woman who did not have a history of allergy was referred for a bone scan to assess the spread of psoriatic arthritis. Some 12-24 hours after administration of the radiopharmaceutical (Medronate II, Amersham International) an acute episode of vasculitis and erythema multiforme developed. At the time of the injection her skin had been clear, and exacerbation of psoriasis was ruled out. Over the next seven days her condition worsened and she had painful inflamed lesions over $80 \%$ of her body, but her domestic circum- stances precluded admission to hospital. Accordingly, she was treated with a topical corticosteroid (clobetasol propionate twice daily) and seen every second day for two weeks. Her condition responded slowly, requiring treatment for four weeks.

The radiopharmaceutical had been prepared from a multidose phial, from which doses for seven other patients were drawn; none of these other patients developed a reaction. Tests for pyrogens and for sterility yielded negative results, and the manufacturer's quality control records did not show any abnormality. The scans obtained two to three hours after injection showed a normal biodistribution of the radiopharmaceutical in all eight patients, with no uptake in the thyroid. Hypersensitivity to sodium medronate was diagnosed on the basis of the symptoms and their time course.

\section{Comment}

Adverse reactions to radioactive diphosphonates, though rare, are the most common type of reactions in nuclear medicine in the United Kingdom, with an incidence of 4-50/100000.' The medical assessor of the British Institute of Radiology, to whom adverse reactions to radiopharmaceuticals are reported, states that most reactions to diphosphonates are reactions to sodium medronate (D Keeling, personal com- 